\title{
La primera visita de Alfonso XIII a la Residencia de Estudiantes
}

Isabel Pérez-Villanueva Tovar*

El domingo 19 de febrero de 1911, Alfonso XIII visitaba por primera vez una joven institución, la Residencia de Estudiantes, nacida del impulso de la Junta para Ampliación de Estudios e Investigaciones Científicas, e inspirada, por tanto, de forma discreta e indirecta, aunque inequívoca y firme, en el ideario de la Institución Libre de Enseñanza. Pieza singular en el sistemático y global proyecto de reforma trazado por el núcleo institucionista a través de la Junta, había sido creada por Real Decreto el 6 de mayo de 1910, e inaugurada, al comenzar el curso siguiente, en la casa número 14 de la madrileña calle de Fortuny. La Residencia de Estudiantes era todavía entonces para sus responsables el "primer ensayo" ${ }^{1}$ - minuciosamente meditado y cuidadosamente preparado, desde luego- de un "pequeño colegio universitario» concebido como «tímido y callado intento hasta ver si la opinión española estaba preparada para recibirlo».

Esta temprana relación del Rey con la naciente institución - lo que su presidente, Alberto Jiménez Fraud, ha llamado «un feliz "incidente"»- contribuyó sin duda a "atraer muy pronto la atención pública sobre el nuevo colegio" ${ }^{2}$. Con tal motivo, el diario $A B C$ da cuenta inmediata y muy atinada del planteamiento y de los fines del centro, "primera célula de un vasto organismo" que quiere ofrecer a los estudiantes uni-

* UNED.

- Junta para Ampliación de Estudios e Investigaciones Científicas, Memoria correspondiente a los años 1910 y 1911, Madrid, 1912, pág. 214.

2 JIMÉNEZ, A., Ocaso y restauración. Ensayo sobre la Universidad Española Moderna, México DF, El Colegio de México, 1948, págs. 218-219 y 222. 
versitarios cuyas familias no residen en Madrid -incluidos aquellos dedicados ya sólo a "trabajos de ampliación e investigación»- «un hogar donde, bajo un régimen de amplia libertad, encuentren ambiente puro de vida sana, simple y honrada, influjos espirituales elevados, estímulos de trabajo, aires de tolerancia y respeto mutuo, ejemplos de buenas maneras, ocasión de goces honestos, dirección y ayuda para sus estudios y elementos para reforzar su cultura general humana, al mismo tiempo que hacen su preparación profesional y especial»».

La misma fuente pone de manifiesto con igual acierto el propósito último del nuevo organismo, formar "hombres", "ciudadanos", y no meros "científicos aislados", esto es, lo único a que podía aspirar "cuando más" la Universidad "tal como la conformó el siglo XIX". En óptima sintonía con las intenciones de los inspiradores y responsables de la Residencia, la reseña subraya también la existencia de una tendencia de «todos los países» en tal sentido, una tendencia a la que se asigna un cuarto de siglo de antigüedad y que supone una sugerente síntesis entre tradición y modernidad: «Todo el mundo volvió la vista a las viejas instituciones que aún vivian en algunas Universidades, como Oxford y Cambridge. $Y$ de ahí arranca un movimiento que tiende a substituir con creaciones modernas aquella acción de los antiguos colegios». Si en 1910 ya existían "casas de residencia para estudiantes» de este tipo en numerosas Universidades inglesas, australianas y norteamericanas, en cada lugar este «movimiento» general tenía que adoptar «el carácter más en armonía con el fondo del alma nacional»; en España, parecía necesario "proceder con prudencia y por vía de ensayo", fiando el logro de la ardua empresa a «la constancia, la paciencia y el esmero». Todo un resumen, ajustado y exacto - tanto que resulta lícito atribuirlo de manera bastante segura a alguno de los más inmediatos impulsores del centro-, del contenido, del alcance y de la forma de actuar de la Junta y de la propia Residencia de Estudiantes.

En aquella inicial y solemne ocasión, Alfonso XIII fue recibido en la sede residencial, no sin protocolo, «por los señores ministro y subsecretario de Instrucción pública, por el Sr. Cajal, presidente de la Junta; el Sr. Menéndez Pidal, presidente del Comité de la Residencia; el señor marqués de Palomares de Duero, D. José Castillejo y otros vocales de aquel Comité. Acompañaron a S.M. en su visita el señor marqués de la Torrecilla, el señor gobernador civil y otras personas". El diario $A B C$ deja constancia de la activa participación en el acto del Secretario de la Junta, José Castillejo — «alma de la Residencia», fue él quien explicó al Rey el funcionamiento de la institución, "demostrando con la mayor sencillez una cultura asombrosa»-, y del talante receptivo de Alfonso XIII: "Su 
Majestad visitó detenidamente la casa, conversó durante una hora, con su acotumbrada afabilidad, con los estudiantes y con las personas citadas, se enteró con todo detalle de los antecedentes de la obra y de las condiciones en que se desarrolla, hizo observaciones que indican cuánto se preocupa y cuán intensamente está penetrado de las modernas orientaciones pedagógicas y ofreció generosamente su concurso para la extensión de este movimiento, que comprende en todo su alcance" ${ }^{3}$.

Alberto Jiménez Fraud, al recordar este primer contacto del Rey con el centro residencial, en presencia de «la plana mayor de la Junta», del entonces Ministro de Instrucción Pública y Bellas Artes, Amós Salvador, y de "altos funcionarios palatinos», destaca, por su parte, que la visita se prolongó, con «cierto asombro de los elementos oficiales», por expreso deseo de Alfonso XIII, quien "con curiosidad mal contenida quería saber lo que había dentro de aquel germen" ${ }^{4}$. Y una Memoria de la Junta, describiendo la vida de la Residencia en ese período, menciona que el Rey "se dignó visitarla, mostrando un sincero interés y ofreciendo generosamente su protección para esta obra" ${ }^{5}$.

Una carta de José Castillejo a su familia fechada el 20 de febrero de 1911, el día siguiente de este acontecimiento, permite seguir de manera viva y detallada sus preparativos, su desarrollo y las reacciones del Rey: «Por lo pequeño del local acordamos no avisar a nadie, y sólo estábamos allí esperando Cajal, Menéndez Pidal, el Marqués de Palomares y yo -escribe el Secretario de la Junta-. En esto llegaron una bandada de individuos de la policía secreta, vestidos de paisano y pretendieron entrar. Nosotros no queríamos; pero Cajal dijo que los dejáramos porque es costumbre y por quitarnos responsabilidad en caso de un atentado o accidente. Allá se metieron y se escondieron en los rincones y en los pasillos. Cajal, como es un poco sordo, decía con voz destemplada "Vienen bien disfrazados. Parecen personas decentes. Unos toman aire cándido, otros de hombres de ciencia". Verdaderamente son innobles. Tuvimos que obligarles a que se quitaran el sombrero, pero no pudimos evitar que tirasen puntas de cigarro y ensuciaran el jardín." Prosigue: «A poco llegó el Ministro de Instrucción Pública y su hijo, el Subsecretario y

\footnotetext{
6.

${ }^{4}$ JIMÉnEZ, A., Ocaso y restauración, op. cit., pág. 222. En esta obra, escrita después de la guerra, Alberto Jiménez Fraud sitúa erróneamente la primera visita de Alfonso XIII al centro residencial el 11 de febrero de 1911.

5 Junta para Ampliación de Estudios e Investigaciones Científicas, Memoria correspondiente a los años 1910 y 1911, op. cit., pág. 211.
}

3 «El Rey en la Residencia de Estudiantes», $A B C$, lunes 20 de febrero de 1911, pág. 
el Gobernador Civil. Y unos instantes después se presentó el Rey en un automóvil, con el Marqués de la Torrecilla. Cajal le saludó y nos presentó a los que estábamos diciendo que yo había sido el organizador de aquello y sería su cicerone. El Rey contestó que ya el Ministro le había hablado de mí y deseaba conocerme, etc..."

Seguidamente, Alfonso XIII fue invitado a entrar en la Biblioteca, donde se le expusieron «los antecedentes de la obra; el movimiento que se nota en todos los países, la propaganda que hacen los alemanes para evitar la mala vida de los estudiantes, la corriente en Inglaterra, en Australia y en los Estados Unidos para fundar casas de estudiantes cuyo objeto es hacer hombres y caballeros y ciudadanos, sin descuidar el lado intelectual y sin utilizar como medios sino la libertad y el ejemplo, y el ambiente y la atracción de las cosas nobles". El Rey fue asimismo informado del régimen interior de la Residencia, de la carencia de «reglamento» y del voluntario rechazo de «otras normas» que no fueran «las exigidas en un hogar o en una familia entre gentes decentes y educadas». Recibió un buen ejemplo del sutil, flexible, pero infalible, método adoptado; porque, al plantearle los planes para consolidar y desarrollar en el futuro el organismo residencial - construir más pabellones para estudiantes, e incluso otros para profesores y "muchachos" casados, que se esperaba contasen con un ingrediente añadido, «la gracia y el calor de la mujer»-, escuchó la aseveración que transcribe José Castillejo en los siguientes términos: "que haríamos comedores separados y una sala de fiesta. Digo de fiestas, añadí, para que no se asusten estos chicos, porque en realidad será para conferencias, proyecciones y trabajos".

Alfonso XIII recorrió toda la casa «y se fue enterando y preguntando por todo"; incluso "se entretuvo en mirar las fotografias de los chicos que éstos tenían en sus cuartos, las camas, armarios, etc." Ya que si, nada más llegar, se le hizo observar cómo se había amueblado la casa "comprando mesas baratas de pino, de la calle de los Estudios pintándolas y adecentándolas", él debió de percibir pronto la peculiaridad de aquel marco ambiental, e intuyendo quizá su auténtico alcance, su significación profunda - el atractivo y riguroso acuerdo entre contenido y continente, de raíz krausista-, "no cesó de repetir que le daba pena decir que no le parecía estar en España al ver una casa tan limpia y tan modesta y tan nueva en su concepción".

En el comedor se le ofrecieron después "dulces y vino" -la víspera el mismo Secretario de la Junta había también encargado «en casa de Lhardy unos fiambres"-; el Rey se sentó "y pegamos de nuevo la hebra charlando en broma -precisa Castillejo-. Le dije que aquel comedor estaba sin terminar porque queríamos ponerle un friso de azulejos y pin- 
tarlo, pero que para dirigir la pintura esperábamos a Sorolla. Se rió mucho y contestó que era preciso echarle mano en cuanto vuelva de América». Ante otra indicación acerca del ajuar - los "cacharros" de Talavera en que se servían los dulces - y acerca del mobiliario, hecho en Madrid con «modelo" meticulosamente ideado por los responsables de la Residencia, «dijo que en España podrían hacerse tantas cosas buenas; pero que está dormida y medio muerta».

Para asegurarse de que Alfonso XIII comprendía bien la singularidad, el valor, la fuerza renovadora de esta clara, sobria y refinadísima realidad material -expresión acabada del propio pensamiento de la Institución Libre de Enseñanza, de su talante, de su proceder, y, más obviamente, de su depurada opción estética-, Castillejo le contó su conversación con el príncipe Kropotkin, sin dejar de advertir previamente que no era «muy reverente su mención en este sitio»: el "gran apóstol de los anarquistas" había manifestado «que no era Inglaterra, sino España la tierra de los caballeros, cuando se ve en el tren ofrecer a los aldeanos la bota y la merienda. Nuestros aldeanos, le dije, son un material de primera fuerza si se los elevara y educase. "No los cambio por ningunos" contestó". En esta misma línea, el Secretario de la Junta transmitió al ilustre visitante cómo «dos Doctoras inglesas» habían alabado ante él «la generosidad de nuestros aldeanos que no querían cobrarles la comida». Valoración, por tanto, de lo autóctono, de lo «popular», en sus rasgos más veraces, más "naturales", adoptando una perspectiva europea, y más en concreto inglesa, con un modelo humano - el gentleman - cuyas cualidades no se vinculan precisamente al sector social que convencionalmente, sólo de forma supuesta, las detentaba. Una magnífica síntesis de una de las claves más importantes del coherente y global proyecto reformista de los impulsores de la Residencia - la armonización entre lo foráneo escogido y lo español más genuino y auténtico-, un sustancioso anticipo también de las "virtudes" individuales y sociales y de su consiguiente conjunción, que, según esta óptica, debía orientar la vida privada y pública. En suma, una intencionada lección, impartida al aún joven monarca mediante un procedimiento táctico, penetrante y ameno, en el que no falta la anécdota.

Finalmente, ya en el jardín, "vinieron los fotógrafos de periódicos a hacer fotografías - concluye Castillejo-. Me llamó el Subsecretario y me hizo ponerme al lado del Rey. $Y$ volvimos a reanudar la charla, medio en serio medio en broma". Al irse, "se despidió afectuosa y llanamente". El Secretario de la Junta, que no deja de formular en la carta a su familia un esperanzado y muy profesoral juicio al modo institucionista sobre la personalidad de Alfonso XIII — «Es un muchacho mucho más listo que los que le rodean y parece que al fin comienza a enterarse de por donde 
va el mundo. Las coge al vuelo y tiene buen sentido y se le conoce el influjo de sus viajes. Qué habría sido si le hubieran educado como es debido!"-, subraya la "gran intimidad, cordialidad y llaneza" demostradas a lo largo de la visita, "persuadido él de que estaba en un grupo de personas decentes".

El Rey dio al parecer sobradas muestras de apreciar en toda su dimensión el origen, el planteamiento y los fines de la institución residencial; a las reflexivas observaciones hechas en tal sentido por José Castillejo contestó que "conocía algunos colegios ingleses donde ha estado su cuñado", repitiendo más de una vez "su impresión de que no le parecía estar en España al ver aquella limpieza y el aire de aquellos chicos». Ante las muy estudiadas explicaciones de su guía, siempre de marcado tono didáctico, sobre la conveniencia de mandar pensionados al extranjero para que después, como «colonizadores», viniesen a «civilizar el país", respondió: "Era absolutamente mi idea». Resaltó también de forma muy tajante que «le parecía bien eso de alternar entre el extranjero y España porque el que vive muchos años fuera luego esto le parece Marruecos (textual) y no puede vivir aquí».

Acerca de la actividad deportiva organizada en la Residencia, manifestó que también él «quería fomentar los juegos y deportes en las Universidades, etc."; dio en este aspecto una prueba evidente de compartir el punto de vista residencial, algo facilitado seguramente por tener en ambos el deporte - signo de distinción social en la España de entonces- un común parangón, el inglés. Así, tras pedir que en las fotografías "le borraran una herida que tenía en la nariz y que le habían hecho jugando al polo", se produjo entre el Rey y Castillejo un breve diálogo, que relata éste con cierta mordacidad: “"Es que jugando, le dije, desaparecen los respetos a la Majestad". "Y si no desaparecieran, me contestó, no sería juego"».

Hablando de «cosas de educación, de lo que estudiaba cada uno de los chicos, del influjo beneficioso de los viajes", y ante una llamada concreta de atención sobre la necesidad de aprender idiomas, reconoció haber sido «muy mal estudiante»: «He estudiado alemán 8 años y no he conseguido aprenderlo, y hasta que presidí el Primer Consejo de Ministros no me enteré de mi ignorancia». Así, el Rey no duda en hacer referencia a su inadecuada educación: «¿Cómo a un chico, me preguntaba, le va a gustar estudiar matemáticas que él no sabe para qué sirven? -escribe Castillejo-. Le contesté que ahora no se enseña ya a nadie matemáticas en las buenas escuelas, sino cuando las necesita, $\mathrm{ni}$ se enseña a leer sino cuando el chico siente gana y estímulo de leer, ni se enseña a escribir sino cuando el chico tiene algo que escribir. En 
América, le dije, han hecho el ensayo de invertir las clases, haciendo que en vez de preguntar el profesor a los alumnos sean éstos los que le pregunten a él. "Lástima, contestó riendo, que se haya descubierto eso cuando yo he acabado ya mis estudios"”.

Algunas expresiones de Alfonso XIII, plasmadas por el Secretario de la Junta, evidencian no sólo su aprobación, sino su resuelta voluntad de apoyar a la naciente institución - «Me dijo que contáramos con él para todo", recalca José Castillejo-, e incluso su intención de facilitar su asentamiento definitivo y su expansión: "me dijo que él se encargaba de gestionar un solar para nosotros. $Y$ al marcharse le dijo a Cajal que él ofrecía construirnos una casa»" ${ }^{6}$.

Además - $y$ así lo puntualiza Alberto Jiménez Fraud-, la recepción del 19 de febrero de 1911 se organizó por deseo personal del Monarca, actuando como intermediario "una persona notoria en la vida española y alejada de la política", el pintor Joaquín Sorolla, que tenía acceso frecuente a Palacio y, al tiempo, mantenía estrecha relación con Francisco Giner de los Ríos y con Manuel Bartolomé Cossío ${ }^{7}$. El Rey precisó en la sede residencial, haciéndose eco de unas palabras del Ministro Amós Salvador sobre lo desconocida que era aún la Residencia a pesar de ser tan «importante y nueva", que él se había enterado de su existencia por «una casualidad», a través del Marqués de la Vega Inclán: "Es que, le contesté yo —cuenta Castillejo-, el Marqués de la Vega Inclán es un calavera y le gusta a veces andar entre estudiantes, y hace pocos días comió aquí con ellos en el sitio donde V.M. se sienta". El se rió y segui-

${ }^{6}$ Carta de José Castillejo a su familia fechada en Madrid el 20 de febrero de 1911, reproducida en Palacios Bañuelos, L., Castillejo, educador, Ciudad Real, Diputación Provincial de Ciudad Real, 1986, págs. 66-70. Ramón Carande aporta una versión chispeante de la anécdota de Kropotkin que Castillejo contó al Rey, si bien parece situarla en el verano de 1914; así, al explicarle el Secretario de la Junta, en una visita realizada a su casa londinense con el joven Carande, que la corporación esperaba que los pensionados que mandaba a Inglaterra aprendiesen a "ser gentlemen", la "réplica del príncipe resonó como el chasquido de un trallazo: - ¡Ah!, claro está; ahora me lo explico, me explico la impresión que me causaron, en mis viajes, en vagones de tercera de los ferrocarriles españoles, lentos y sucios; los aldeanos y otros pobres castellanos que nos ofrecian sus provisiones, a la hora de comer, y ayudaban a mi hija a descender del tren y la acompañaban en el andén, cuando ella quería pasearse. Yo no podía imaginar que aquellos viajeros estuvieran educados en Londres". La "desmedida anglofilia" de Castillejo, "desplegada delante de quien no la compartía", explica tal "desliz", «inconcebible - según Ramón Carande- en quien no admiraba menos las inveteradas tradiciones de nuestro pueblo" (CARANDE, R., Galería de raros atribuidos a Regino Escaro de Nogal. Prólogo de R. Pérez Delgado, Madrid, Alianza, 1982, págs. 41-42).

7 JiMÉNEZ, A., Ocaso y restauración, op. cit, pág. 222. 
mos de broma recordando lo mucho que el Marqués de la Vega Inclán ha hecho ya por España» ${ }^{8}$.

Este primer y temprano acercamiento de Alfonso XIII a la Residencia de Estudiantes creada por la Junta para Ampliación de Estudios e Investigaciones Científicas parece formar parte de un proyecto politico de envergadura y debe inscribirse, por tanto, en un marco de referencias que supera la mera experiencia residencial. $Y$ ello por cuanto supone en realidad una aproximación de la monarquía a la labor emprendida por la Junta en 1907, lo que a su vez significa establecer contacto con el planteamiento reformista y liberal que ésta plasma con perspectiva global a través de sus realizaciones, todas ellas diversas pero complementarias. De hecho, esta visita del Rey al centro residencial hizo que se atribuyeran a la recién fundada institución «intenciones y planes políticos»: "Contemplaba en aquella fecha la monarquía una mayor aproximación a instituciones y hombres liberales -resume Jiménez Fraud-. Personas cercanas al Rey creían ver en las actividades de la Junta para Ampliación de Estudios y en la creación de la Residencia —obras que sabían eran fruto de la labor reformista de la Institución-, como movimientos de avanzada de una amplia corriente nacional dispuesta a emplear métodos de tolerancia y colaboración y de autorizada información en un tratamiento liberal y progresivo de los negocios del Estado. $Y$ apoyándose en lo que estimaban un estado de opinión en proceso de crecimiento, deseaban que el Jefe del Estado tomase contacto con las nuevas instituciones" ${ }^{9}$.

Al visitar la Residencia de Estudiantes, Alfonso XIII se acercaba a lo que Carlos Seco Serrano ha llamado «las más sazonadas reservas intelectuales del país - las que se canalizaban en la Institución Libre de Enseñanza-" ${ }^{10}$, movimiento con hondo significado político de aproximación entre la intelectualidad republicana y la monarquía, que, iniciado en tiempos de José Canalejas, alcanzaría su momento culminante - y sus expresiones más visibles- al aceptar entrevistarse con el Rey, en el Palacio Real, Gumersindo de Azcárate e incluso Manuel Bartolomé Cossío, además del Presidente de la Junta para Ampliación de Estudios e Investigaciones

${ }^{8}$ Carta de José Castillejo a su familia fechada en Madrid el 20 de febrero de 1911 , op. cit., pág. 68.

9 JiMENEZ, A., Ocaso y restauración, op. cit., pág. 222; véase también pág. 186.

10 Seco Serrano, C., Alfonso XIII y la crisis de la Restauración, Barcelona, Ariel, 1969, pág. 102. 
Científicas, Santiago Ramón y Cajal, en un momento, enero de 1913, en que el Gobierno estaba ya presidido por el Conde de Romanones ${ }^{11}$.

Naturalmente, Alfonso XIII sabía que existía relación entre la Residencia de Estudiantes y la Institución Libre de Enseñanza; pocos días antes de ser recibido en el centro residencial había hecho incluso «un intento" de visitar la sede institucionista del Paseo del Obelisco 8, lo que Francisco Giner rechazó "con dureza que a algunos pareció extremada». Según relata Jiménez Fraud, "cuando S.M. insistió en que llamaría a casa de Giner, éste respondió al amigo oficioso: "La Institución tiene dos puertas, y cuando S.M. nos haga el honor de llamar a una de ellas, yo saldré por la otra" ${ }^{12}$. No cabe duda de que la Institución Libre de Enseñanza, corporación estrictamente privada, supo preservar "cuidadosamente intacta la pureza de su ley y la íntima modestia de su origen ${ }^{13}$, sin confundirse $-y$ procurando también que no se la confundiera, por razones, entre otras, de orden estratégico- con aquellos organismos de carácter público como la Junta y sus derivaciones, que tan hábil y fecundamente había hecho prender en la anquilosada Administración española.

La percepción del Rey -como, por lo demás, la de buena parte de la opinión pública, incluidos los sectores políticos frontalmente enfrentados a cualquier tipo de perspectiva liberal- no era del todo exacta, al identificar sin matización alguna la Institución con la Junta, y, por tanto, con la Residencia. Tan es así que el 19 de febrero de 1911, durante la estancia en la sede residencial, Alfonso XIII volvió a reiterar nuevamente, como recoge Castillejo, su intención de ir personalmente a la casa institucionista: "dijo que ahora deseaba conocer la Institución Libre de Enseñanza porque sabe que allí es donde mejor se enseña. Le contestamos que era un centro particular y el Ministro le ofreció, sin embargo, llevarle a verlo" ${ }^{14}$.

Las coordenadas políticas en las que se produce esta "primera visita regia del año 11» explican «el profundo sentido» de la «breve escena» protagonizada a continuación por Francisco Giner: "Después de salir el regio huésped, y más tarde los demás visitantes, y vuelto el pequeño

11 Véanse, por ejemplo, Ibidem, págs. 97-98, AzcáRATE, P. DE, Gumersindo de Azcárate. Estudio biográfico documental. Semblanza. Epistolario. Escritos, Madrid, Tecnos, 1969, pág. 108, y Fernández Almagro, M., Historia del reinado de D. Alfonso XIII, Madrid, Sarpe, 2 t., 1986, t. I, págs. 184-186.

12 JIMÉNEZ, A., Ocaso y restauración, op. cit., págs. 223-224.

13 Xirau, J., Manuel B. Cossio y la educación en España, Barcelona, Ariel, 2. ed., 1969, pág. 56.

14 Carta de José Castillejo a su familia fechada en Madrid el 20 de febrero de 1911, op. cit., pág. 68 . 
colegio a su discreto silencio, me entregaron una pequeña nota en que Giner me decía que estaba allí cerca, en la Castellana, y que deseaba verme", recuerda Jiménez Fraud. "Salí al paseo y al ver a don Francisco noté que le dominaba una emoción. Le alargué la mano, y estrechándomela con fuerza, exclamó: ¡Criatura, qué responsabilidad!» Patente muestra de la vigilante, aunque solapada, intervención de Giner en las actividades residenciales - sólo entró en la Residencia en una ocasión, "perdido entre el público», para escuchar una conferencia de Unamuno-, tal circunstancia, sobre todo si se vincula la visita del Rey al centro con la prácticamente simultánea negativa que recibe su solicitud de acceder a la sede institucionista, manifiesta que la notable diferencia existente entre los organismos de la Junta y la Institución Libre de Enseñanza era conscientemente asumida por sus responsables e inspiradores.

Ello implicaba a su vez dos formas bien diferentes de actuación respecto al poder público, y más concretamente en este caso respecto a la monarquía; analizando las palabras de Giner -las que pronunció con ocasión de su encuentro en la Castellana y aquellas otras que dijo al reiterar Alfonso XIII su voluntad de ser invitado a la casa de la Institución--, escribe el Presidente de la Residencia de Estudiantes: "era demasiado fuerte Giner para ser juego de veleidades personales, pero el Jefe del Estado era la representación oficial de éste, y el honor que regalaba a la modesta y apenas nacida Residencia había que devolverlo con inmenso esfuerzo para ser digno de él». Para despejar la aparente contradicción, concluye: «Nos separamos en silencio, y yo me alejé pensando, no sé por qué asociación de ideas, en una frase que con frecuencia repetía Giner, y era que la revolución había que hacerla en los espíritus y no en las barricadas, frase que en un ensayo suyo ampliaba en esta forma: "por su trágico aparato, las revoluciones imponen y amedrentan, y nos parecen que trituran las entrañas del mundo, cuando apenas arañan la superficie" $"{ }^{15}$.

Si esta «asociación de ideas» de Jiménez Fraud ilustra a la perfección la modalidad de "revolución" que emprendió el núcleo institucionista, la astuta práctica que se adoptó para llevarla a cabo se descubre con claridad en la defensa de la Residencia que hicieron, ante el Congreso, dos Diputados tan incondicionales de la Institución Libre de Enseñanza como Eduardo Vincenti y Hermenegildo Giner de los Ríos. La

15 JIMÉNEZ, A., Ocaso y restauración, op. cit., págs. 223-224. La presencia de Giner en la Residencia de Estudiantes para escuchar, en torno a 1914, a Unamuno queda confirmada con el testimonio de Antonio J. ONIEVA, "Recuerdos de la Residencia (Fragmentos)", Revista de Occidente, 2. ${ }^{a}$ ép., VI, 66 (julio-septiembre 1968), pág. 300. 
primera visita del Rey al centro residencial constituyó allí, y por tanto ante la opinión pública, una baza importante para asegurar la continuidad y el pleno desarrollo de la Residencia, e incluso, de forma más indirecta, para contrarrestar las críticas que en mayor medida arreciaron contra la Junta y algunas de sus restantes iniciativas; esgrimiéndola como bandera, se consiguió por lo pronto una imprescindible subvención para la institución residencial en los presupuestos de 1913.

Durante su discusión parlamentaria, el día 20 de noviembre de 1912, con el fin de responder al frontal ataque que acababa de recibir la Junta, evocó Vincenti la labor de la Residencia en términos, como es lógico, muy elogiosos, y además tranquilizadores para los inmovilistas, para los moralistas al uso - justamente los sectores que de forma más virulenta y sostenida quisieron impedir la acción conjunta de aquella corporación-, para «la opinión conservadora que se asustaba fácilmente», en definición de José Castillejo, para «los intolerantes católicos que estaban inquietos ante la posibilidad de que el influjo de ideas extranjeras y la libertad de pensamiento pusiesen en peligro la unidad religiosa y la dirección de la Iglesia, que ellos consideraban como derechos de la mayoría» ${ }^{16}$; la describió como modelo indiscutible para propios y extraños, poniendo buen cuidado en resaltar especialmente las alabanzas que de ella había hecho Alfonso XIII: «da a los padres lo que más desean -dijo-: tranquilidad para llevar alli a su hijos en lugar de hacerlo a las casas particulares, donde no tienen ni la luz ni el ambiente que hay en aquella Residencia de estudiantes, donde hay una libertad prudente, porque se trata de personas hechas, de adultos; donde hay cursos de idiomas gratuitos; y lo que ha pasado es que los extranjeros que han venido al Congreso del turismo y han ido a la Residencia de estudiantes han dicho que es la institución mejor de toda Europa, incluso mejor que el dormitorio de la Universidad de Harvard, y el Jefe del Estado ha ido a visitar aquella casa y ha visto aquel "confort", aquel arte, aquella limpieza, que es la cosa más difícil, aquí donde no tenemos la delicadeza de sentimientos, ni gusto artístico, y el Jefe del Estado ha dicho que era lo único europeo que había visto" ${ }^{17}$.

El 23 de noviembre, Hermenegildo Giner de los Ríos, para neutralizar la «verdadera obsesión» existente a su juicio contra el centro residencial -Eloy Bullón, Diputado de la minoría conservadora, había hablado tres días

${ }^{16}$ Castillejo, J., Guerra de ideas en España. Filosofía, política y educación. Prólogo de J. Caro Baroja. Introducción de M. E. Sadler. Traducción de M. de Ferdinandy, Madrid, Revista de Occidente, 1976, págs. 111 y 115.

${ }^{17}$ Diario de Sesiones del Congreso, 20 de noviembre de 1912, pág. 5.285. 
antes contra el hecho de que fuese totalmente independiente de la Universidad—, efectuó la siguiente intervención: «yo no me atrevo a elogiarlo demasiado, porque he tenido un hijo en esa Residencia de estudiantes. $Y$ no quiero que se atribuya a reconocimiento lo que es justicia debida. Pero séame permitido repetir el elogio que salió de augustos labios; frase de encomio de tal naturaleza, que dijo que conocía muy pocas cosas en su propio pais que estuviesen a la altura de aquella Residencia de estudiantes; y hombres de todos los partidos y de toda condición social alaban con entusiasmo la labor de un joven tan modesto como ilustrado, tan honorable como inteligente, tan correcto como enérgico, que rige aquella naciente institución con el espíritu de quien cumple un sacerdocio, y al que es preciso tributar todo el aplauso que se merece" ${ }^{18}$.

Elocuentes ejemplos de la prudente, segura, sabia -y pragmáticaestrategia con que se orientó desde sus inicios la vida de la Residencia de Estudiantes - y de la Junta en su conjunto-, una estrategia que contaba también con las propias «virtudes», con el «tacto» - cualidad tan institucionista- de su Presidente; un reflejo sintomático del difícil y original equilibrio del discurrir institucionista en la esfera de la Instrucción Pública: mantener su horizonte espiritualista y transcendente, sin traicionar ni su meta ni su ímpetu reformistas -intimamente acordes en este ámbito por el sugestivo acuerdo existente entre fondo y forma, teoría y práctica, pensamiento y acción, razón y sentimiento-, pero sin naufragar tampoco en los cambiantes y peligrosos torbellinos políticos y sociales de la realidad española de su tiempo.

Desde este punto de vista, el temprano contacto del Rey con la Residencia de Estudiantes tuvo una dimensión importante, más allá de aquel rápidamente frustrado acercamiento entre la intelectualidad republicana y la monarquía, más allá, por supuesto, del inmediato logro de una concreta asignación económica. Al margen de la evolución política, Alfonso XIII, junto a otros miembros de la familia real como la Infanta Isabel, o, sobre todo, la Reina Victoria Eugenia -atraída quizá de forma particular por el muy claro eco inglés del centro-, visitaron repetidas veces la Residencia de Estudiantes.

Según Alberto Jiménez Fraud, mucho menos optimista que José Castillejo al enjuiciar, ya en 1948, la reacción del Rey el 19 de febrero de 1911 — «no pude averiguar la impresión que le produjeron las res-

${ }^{18}$ Diario de Sesiones del Congreso, 23 de noviembre de 1912, pág. 5.364. El Diputado se refiere en la intervención a su hijo, el arquitecto Bernardo Giner de los Ríos, miembro en época temprana de la Residencia de Estudiantes. 
puestas de Cajal, Menéndez Pidal y el Presidente de la Residencia", escribe-, el Monarca mantuvo siempre una posición ambivalente respecto a la institución: «en los veinte años que se prolongó su reino demostró una confianza grande en la disciplina moral y en el tono elevado de la nueva institución. Siguió acudiendo a ella con extremada frecuencia y actitud mixta de confianza entregada y reserva crítica, pero sin animosidad, aunque tratasen infructuosamente de despertarle esta última los enemigos tradicionales, cuya obcecada imaginación veía ya en los nuevos Residentes enemigos tan "peligrosos" como los secuaces de los diferentes "ismos", a los que habían jurado una guerra a muerte". Nunca abandonó "una última actitud de reserva» hacia la Residencia, a pesar de que «el largo contacto con ella y el invariable respeto y agradecimiento con que se recibían sus visitas le aseguraban de que no perseguíamos ninguna obra perjudicial al régimen y que estábamos siempre dispuestos a cualquier colaboración francamente constructiva».

$Y$, sin embargo, la Residencia de Estudiantes consiguió en buena medida contrarrestar la influencia de esos «enemigos tradicionales" mediante la muy estrecha proximidad al Rey de algunos de sus defensores más entusiastas; porque, hay que subrayarlo, «gran parte de los más allegados colaboradores de la Residencia pertenecían a círculos íntimos palatinos". La alerta inteligencia, la fina sensibilidad, el preciso tino de su Presidente, que tanto contribuyeron a asegurar y reforzar en el entramado residencial la pronta sagacidad de Castillejo en la configuración y en la marcha general de la Junta, jugaron también un papel sobresaliente en la formación de una tupida, firme, infranqueable red de apoyos individuales de diverso signo político y social en favor de la Residencia. Los acérrimos «enemigos tradicionales" del fructífero reformismo liberal de la Institución Libre de Enseñanza no consiguieron sobrepasar, en el terreno de la Residencia de Estudiantes, la definitiva "resignación», con algún guiño de socarrona complicidad, de Alfonso XIII: «Acudía, pues, siempre con absoluta seguridad moral, con curiosidad y con alguna indicación o broma preparada que pudiera perturbar mi ecuanimidad -observa Alberto Jiménez Fraud al rememorar las visitas regias al centro-. Pero mi aire fácil y amable, alejado de todo moralismo o pedantería, y de apariencia siempre flexible, ganaba su simpatía y creo que le hizo llegar a la conclusión de que después de todo valía más respetar la orientación algo "original" de aquella casa»"

19 JIMÉNEZ, A., Ocaso y restauración, op. cit, págs. 222-223 y 259. 